\title{
EM]
}

\section{Supporting research and development in ambulance services: research for better health care in prehospital settings}

A Niroshan Siriwardena, Rachael Donohoe, John Stephenson, et al.

Emerg Med J 2010 27: 324-326

doi: 10.1136/emj.2009.072363

Updated information and services can be found at:

http://emj.bmj.com/content/27/4/324.full.html

These include:

References This article cites 4 articles, 1 of which can be accessed free at: http://emj.bmj.com/content/27/4/324.full.html\#ref-list-1 $\begin{gathered}\text { Email alerting } \\ \text { service }\end{gathered}$
$\begin{gathered}\text { Receive free email alerts when new articles cite this article. Sign up in the } \\ \text { box top right corner of the online article. }\end{gathered}$ service

Notes

To order reprints of this article go to:

http://emj.bmj.com/cgi/reprintform

To subscribe to Emergency Medicine Journal go to:

http://emj.bmj.com/subscriptions 


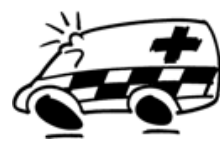

${ }^{1}$ East Midlands Ambulance Service NHS Trust, Lincoln, UK ${ }^{2}$ University of Lincoln, Lincoln, UK

${ }^{3}$ London Ambulance Service NHS Trust, London, UK

\section{Correspondence to} Professor A Niroshan Siriwardena, Faculty of Health, Life and Social Sciences, University of Lincoln, Brayford Pool, Lincoln LN6 7TS, UK nsiriwardena@lincoln.ac.uk

All authors contributed to the article.

Accepted 8 July 2009

\title{
Supporting research and development in ambulance services: research for better health care in prehospital settings
}

\author{
A Niroshan Siriwardena, ${ }^{1,2}$ Rachael Donohoe, ${ }^{3}$ John Stephenson, ${ }^{1}$ Paul Phillips ${ }^{1}$
}

\begin{abstract}
Background This paper discusses recent developments in research support for ambulance trusts in England and Wales and how this could be designed to lead to better implementation, collaboration in and initiation of highquality research to support a truly evidence-based service.
\end{abstract}

Method The National Ambulance Research Steering Group was set up in 2007 to establish the strategic direction for involvement of regional ambulance services in developing relevant and well-designed research for improving the quality of services to patients.

Results Ambulance services have been working together and with academic partners to implement research and to participate, collaborate and lead the design of research that is relevant for patients and ambulance services.

Conclusion New structures to support the strategic development of ambulance and prehospital research will help address gaps in the evidence for health interventions and service delivery in prehospital and ambulance care and ensure that ambulance services can increase their capacity and capability for high-quality research.

\section{INTRODUCTION}

Ambulance services have recently developed new structures to support the growing agendas of prehospital research (research in clinical settings located between primary care and acute services), effectiveness and quality improvement. These structures have developed through strong leadership and a recommendation for local as well as national research activity and research capacity development. ${ }^{1}$

As a result, the National Ambulance Research Steering Group was set up in 2007. It comprises research leads from the regional ambulance services in England and Wales as well as representatives from other key groups involved in prehospital research. The aim of the group is to support the strategic development of ambulance and prehospital research and to seek active involvement, ownership, participation and collaboration with trust boards and executives and academic partners.

The national coordination and support for research has begun to enable sharing of best practice, collaboration and coordination of effort to raise the profile of research in ambulance services. As well as facilitating implementation of research findings through improved access to knowledge, it has also begun to measure and develop research capacity in ambulance services and is seeking to promote academic institutions and groups as research partners, thereby promoting multicentre and even international studies.

\section{BACKGROUND}

The national health research strategy Best Research for Best Health, ${ }^{2}$ the evidence required to underpin the transformation of emergency services as set out in Reforming Emergency Care $^{3}$ and, more recently, Taking Health Care to the Patient ${ }^{4}$ have been key policy drivers for ambulance services research.

Best Research for Best Health seeks "to improve the health and wealth of the nation through research". If its mission "to create a health research system in which the NHS supports outstanding individuals, working in world-class facilities, conducting leading-edge research, focused on the needs of patients and the public" is to succeed in the prehospital arena, this needs to support and be supported by organisational structures for ambulance services research.

In order for prehospital and ambulance research to flourish, these goals have been incorporated into the new structures for nurturing research and development in the newly reconfigured ambulance services in the UK.

\section{INTEGRATED STRUCTURES FOR PREHOSPITAL AND AMBULANCE RESEARCH}

Prehospital and ambulance research, whether in the UK or elsewhere, needs a coherent approach if it is to deliver a vision of patient-orientated world-class research. Ambulance trusts and clinicians will need to develop as users of, collaborators in and initiators of high-quality research to support a truly evidencebased service. This requires a framework that encourages and supports a culture of evidencebased practice, research and innovation, placing ambulance services at the leading edge of patientfocused service delivery. The current structures have been designed to support interaction between knowledge management, quality improvement and research (figure 1).

The key structures are two national groups-the National Ambulance Research Steering Group and the National Ambulance Clinical Audit Steering Group. Both groups have representation from research or audit leads from each of the regional ambulance trusts as well membership from other key stakeholders including Chief Executives and Directors of Clinical Care of ambulance services. Both groups report to the Directors of Clinical Care and therefore are informed by clinical priorities of services as well as understanding the research or 


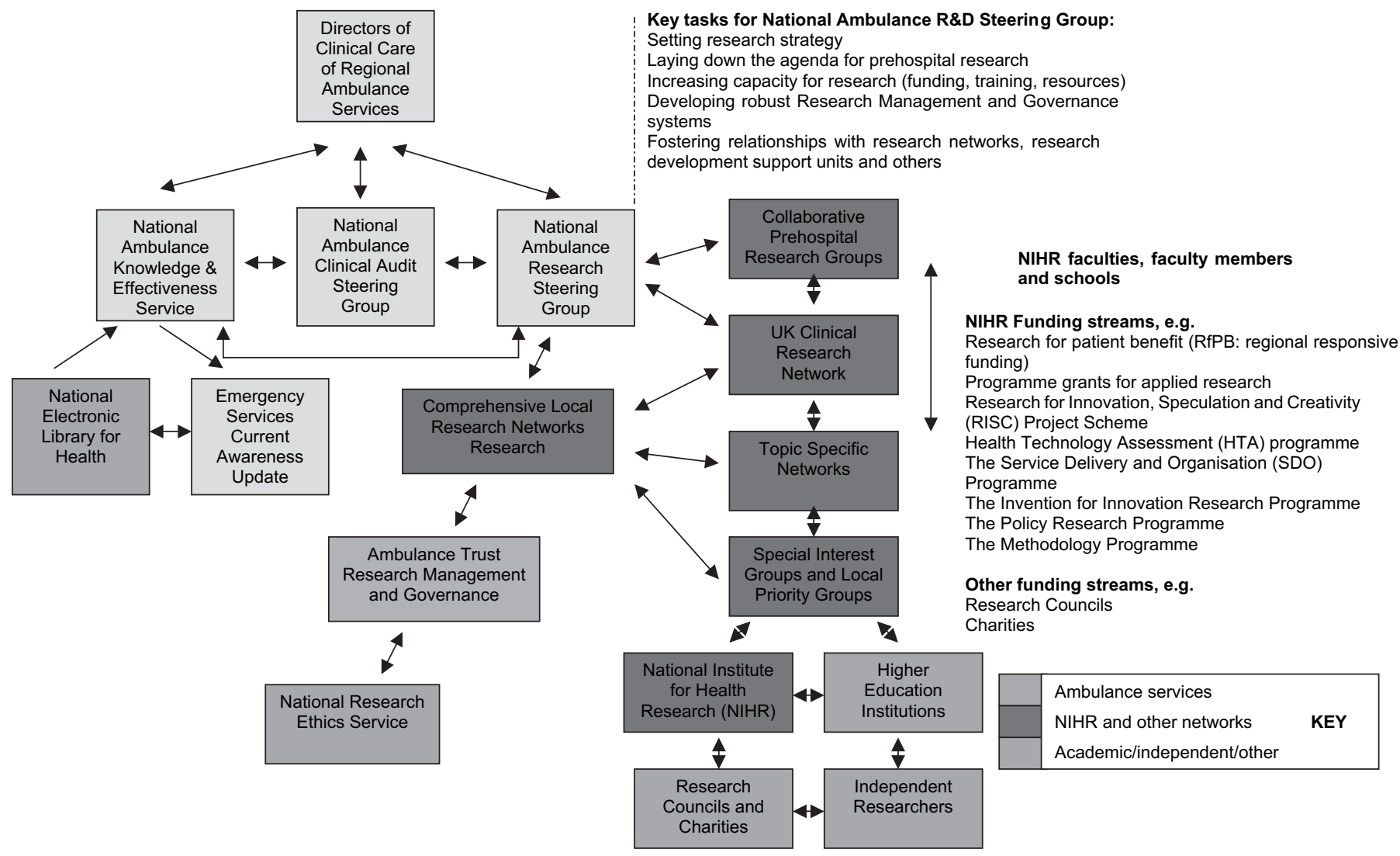

Figure 1 Framework for research and development for ambulance services.

quality improvement agenda. The National Ambulance Knowledge and Effectiveness Service, while not a separate group, does provide a bimonthly clinical effectiveness update (the Emergency Services Current Awareness Update) which is circulated widely to front-line and other ambulances service personnel. Members of the research group also have strong links with the UK Clinical Research Network (UKCRN) and the Comprehensive Local Research Networks (CLRNs), and this has led to new funding to develop the infrastructure in ambulance services for supporting research studies.

The National Ambulance Research Steering Group has sought to promote a sustainable infrastructure capable of identifying and responding to research needs in the short, medium and long term. A number of existing and new national and international research groups have developed to positively support ambulance services research. Collaborative research, including more than one regional ambulance service linked to one or more university departments specialising in prehospital or primary care, has been established in London and Sheffield for many years and has begun to flourish elsewhere-for example, in Swansea, Manchester and Nottingham.

One of the earliest academic centres for prehospital research in the UK, the Medical Care Research Unit of the University of Sheffield, was established in 1966 and continues to play a significant role in emergency and urgent care research. The international 999 EMS Research Forum was formed in 1999 to encourage, promote and disseminate research and evidence-based policy and practice in emergency health care. The Thematic Research network for emergency and UnScheduled Treatment (TRUST), funded by the Wales Office for Research and Development in Health and Social Care (WORD), was the first nationally funded research network in emergency and urgent care. The British Paramedic Association Research and Audit Committee was established to offer professional support and coordination and to focus on developing research capacity in the emerging paramedic profession. Other newer groups are also emerging.

True collaboration will help to move ambulance services from organisations which have been traditionally seen as 'data rich, information poor' to bodies which themselves espouse and nurture a culture of research and innovation. It is also important that opportunities are sought for collaborative multidisciplinary research which crosses the interface between primary, prehospital or acute settings.

As well as supporting the current national priority for translational research, the strengthening of links between the triad of research, clinical audit (quality improvement) and knowledge management has the potential to enable the more rapid dissemination of evidence-based practice and the evaluation of new models of service delivery supported by effective knowledge transfer.

The new structures have also worked to ensure that effective research governance procedures are established and maintained. Healthcare organisations which lead or participate in research are required to have systems in place to ensure that the principles and requirements of research ethics and governance are consistently applied and, in common with all NHS organisations, ambulance trusts are required to be compliant with legislation such as the Medicines for Human Use (Clinical Trials) Regulation 2004 for clinical research trials and the research elements of the Mental Capacity Act 2005 before recruiting to relevant studies.

\section{NATIONAL RESEARCH STRUCTURES}

The NHS research strategy is driving the research agenda rapidly forwards in all health sectors. The key structures include the National Institute for Health Research (NIHR), a virtual 
institute which supports clinical researchers in the NHS, the research networks which will support high quality clinical research and research management and governance, and the new and existing funding systems for NHS-funded research.

Ambulance services have been included in configurations for CLRNs, which are administering the new 'bureaucracy busting' processes for research governance as well as providing funding for service support costs to support additional health service costs of participating in clinical research. Ambulance trusts still need to have arrangements-either alone or in partnership with other healthcare organisations-to administer governance arrangements for 'own account' and student research projects which will not be adopted by the CLRNs.

The CLRNs together with the six Topic Specific Research Networks (TCRNs) and a Primary Care Research Network (PCRN) make up the NIHR Clinical Research Network (CRN) in England. This is part of the UKCRN which aims to provide a world-class NHS infrastructure for clinical research, supporting studies adopted by the network and ensuring rigorous governance. The CRN will extend to all aspects of health care including ambulance services, so it is important that ambulance services are represented and active in these networks. More recently, the CRN has set up special interest groups including a group for injuries and accidents as well as local priority groups in this theme linked to CLRNs.

The UKCRN will support adopted studies funded by the NIHR and the research (funding) councils such as the Medical Research Council and Economic and Social Research Council as well as some health research charities. The research funding organisations, along with the Department of Health, will be among those responsible for determining research priorities based on stakeholder and other views. ${ }^{6}$ Ambulance services will need to compete for national and international funding in partnership with other healthcare organisations and higher education institutions. However, the UKCRN and the NIHR will not be a panacea for previous shortcomings in prehospital research or research funding to ambulance services, and strong collaborations between ambulance services and academic departments will be needed to address these deficiencies.

There are an increasing number of examples of collaboration between academic departments and ambulance services in high priority topics such as developing measures linked to patient benefit, providing safe pathways of care not involving transport to hospital, and increasing evidence-based clinical care delivered in the prehospital environment. ${ }^{6}$ Examples of these include national studies on emergency care practitioners (http://www. shef.ac.uk/scharr/sections/hsr/emergency/ecp.html), paramedic management of falls (http://www.controlled-trials.com/isrctn/ pf/60481756), clinical performance indicators ${ }^{7}$ and studies with industry to determine the benefits of prehospital thrombolysis versus primary percutaneous coronary interventions (http:// www.presseportal.de/pm/6631/1043644/boehringer_ingelheim).

The new research structures, by ensuring that ambulance services and academic departments meet regularly together to discuss potential new research studies, have and will continue to facilitate partnerships for large-scale research and quality improvement projects between one or more academic departments and regional ambulance services, thus ensuring sufficiently powered studies across representative populations providing generalisable and relevant research outputs.

\section{DEVELOPING FUTURE CAPACITY}

Research capacity building, the "process of individual and institutional development which leads to higher levels of skills and greater ability to perform useful research", ${ }^{8}$ will be a key factor in producing a sound research culture for ambulance services. ${ }^{6}$

The National Ambulance Research Steering Group is undertaking a research capacity development audit across most of the UK ambulance services in partnership with Trent and South Yorkshire Research Design Services using a previously developed framework. ${ }^{9}$ Organisational support for research, collaboration with academic departments and the development of expertise in accessing funding are some of the critical elements required for this. Research on current priorities ${ }^{6}$ and novel studies, proposed by ambulance services and staff in line with their strategic aims, will enable a research culture to develop talented researchers, resources for individual studies and future programmes of prehospital research in collaboration with academic departments.

\section{CONCLUSION}

This short paper describes how new structures have been put in place to support the strategic development of ambulance and prehospital research. This will help address gaps in the evidence for health interventions as well as service delivery in the rapidly changing environment of prehospital and ambulance care. We believe that this framework for research will develop to support clinicians in providing the best care to their patients, managers to implement the systems for this to occur and commissioners to procure the highest quality services based on the best available evidence. Ambulance services will aspire to be taken seriously as research active and capable organisations.

Acknowledgements The authors acknowledge the support of members of the National Ambulance Research Steering Group.

Funding Department of Health.

\section{Competing interests None.}

Provenance and peer review Not commissioned; externally peer reviewed.

\section{REFERENCES}

1. Siriwardena AN. Raising the game: Nurturing research and development in the newly reconfigured ambulance services in the United Kingdom - opportunities for research for better health in prehospital settings. Nottingham: East Midlands Ambulance Service NHS Trust, 2007.

2. Department of Health Research and Development Directorate. Best research for best health. A new national health research strategy. London: Department of Health, 2006.

3. Department of Health. Reforming emergency care: first steps to a new approach. London: Department of Health, 2002.

4. Department of Health. Taking healthcare to the patient: transforming NHS ambulance services. London: Department of Health, 2005.

5. Peconi J, Snooks H, Edwards A. Thematic Research network for emergency and UnScheduled Treatment (TRUST): scoping the potential. BMC Emerg Med 2008;8:2.

6. Snooks H, Evans A, Wells B, et al. What are the highest priorities for research in pre-hospital care? Emerg Med J 2009;26:549-50.

7. Siriwardena AN, Shaw D, Donohoe R, et al. Development and pilot of clinical performance indicators for English ambulance services. Emerg Med J 2010 (in press).

8. Trostle J. Research capacity building and international health: definitions, evaluations and strategies for success. Soc Sci Med 1992;35:1321-4.

9. Cooke J. A framework to evaluate research capacity building in health care. BMC Fam Pract 2005;6:44. 